\title{
REVIEW
}

\section{New Frontiers in Subcutaneous Immunoglobulin Treatment}

Stephen Jolles · Mark R. Stein · Hilary J. Longhurst • Michael Borte • Bruce Ritchie • Matthias H. Sturzenegger • Melvin Berger

To view enhanced content go to www.biologicstherapy-open.com Received: October 28, 2011 / Published online: 14 December, 2011

(c) The Author(s) 2011. This article is published with open access at Springerlink.com

\section{ABSTRACT}

Subcutaneous immunoglobulin (SCIG) treatment provides stable serum immunoglobulin G (IgG) levels, is associated with fewer systemic adverse events than intravenous immunoglobulin (IVIG) treatment, and offers the convenience of home therapy. In clinical practice, IVIG is still used preferentially for initiation of treatment in newly diagnosed patients with primary immunodeficiency (PI) and for immunomodulatory therapy, such as treatment of peripheral neuropathies, when high doses are believed to be necessary. The authors discuss recent experience in using SCIG in place of IVIG in these settings. SCIG has been successfully used for initiation of therapy in previously untreated PI patients. Seventeen of 18 PI patients achieved serum IgG levels $\geq 5 \mathrm{~g} / \mathrm{L}$ after the loading phase. Daily treatment was well tolerated and provided opportunities for patient/parent training in selfinfusion. SCIG has been used for maintenance therapy in multifocal motor neuropathy (MMN) in three recent clinical trials, with good efficacy and tolerability results. Seven of eight MMN patients maintained serum IgG levels of $14-22 \mathrm{~g} / \mathrm{L}$ with a mean dose of $272 \mathrm{mg} / \mathrm{kg} /$ week, had stable muscle strength, and felt comfortable
Stephen Jolles ( $\square$

University Hospital of Wales, Heath Park, Cardiff, CF14 4XW, UK. Email: jollessr@cardiff.ac.uk

Mark R. Stein

Allergy Associates of the Palm Beaches, North Palm Beach, FL, USA

Hilary J. Longhurst

Barts and London NHS Trust London, London, UK

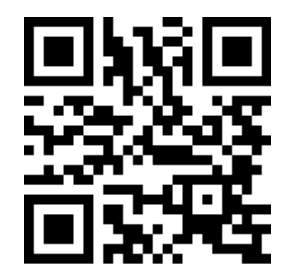

Enhanced content for this article is available on the journal web site: www.biologicstherapy-open.com

\section{Michael Borte}

Hospital "St. Georg" GmbH Leipzig, Academic Teaching Hospital of the University of Leipzig, Leipzig, Germany

Bruce Ritchie

Division of Hematology, Faculty of Medicine, University of Alberta, Edmonton, AB, Canada

\section{Matthias H. Sturzenegger}

Department of Neurology, University Hospital and University of Berne, Berne, Switzerland

Melvin Berger

CSL Behring LLC, King of Prussia, PA, USA, and Case Western Reserve University, Cleveland, OH, USA 
with self-administration. Four patients with polymyositis or dermatomyositis achieved improvement in serum creatine kinase levels and muscle strength with SCIG therapy. Recent experience with SCIG suggests that traditional concepts of immunoglobulin therapy may be challenged to increase available therapy options. SCIG can be used to achieve high IgG levels within several days in untreated PI patients and to maintain high serum levels, as shown in patients with MMN.

Keywords: immunoglobulin G; immunoglobulin therapy; multifocal motor neuropathy; primary immunodeficiencies; serum levels; subcutaneous administration

\section{INTRODUCTION}

Intravenous immunoglobulin (IVIG) has been the preferred route of therapy for primary immune deficiencies since the early 1980s. Subcutaneous immunoglobulin (SCIG) administration was first described by Bruton in 1952. ${ }^{1}$ Later, SCIG administration with small pumps was introduced in the United States (US) and became widely used in Sweden and Norway. ${ }^{2-4}$ Positive treatment experience increased the popularity of the subcutaneous route among physicians and patients elsewhere. ${ }^{5,6}$ SCIG therapy obviates the need for venous access, maintains stable serum IgG levels, offers fewer systemic adverse events (AEs) when compared to IVIG, and is amenable to self-infusion. ${ }^{7-9}$ Self-infusion and home administration allow flexibility in adapting to the patient's own schedule and are associated with improved quality of life compared to hospital or office-based intravenous treatment. ${ }^{10-12}$

Recent IVIG products are still given mostly at a clinic, doctor's office, or infusion center. Recently developed SCIG preparations offer more rapid infusion in addition to good efficacy and tolerability. These technological advancements prompt reconsideration of the use of SCIG in different indications currently reserved for IVIG.

In current practice, SCIG has mainly been used for maintenance replacement therapy in primary immunodeficiency (PI), while IVIG is used for initiation and maintenance of replacement therapy and for the high doses required in immunomodulatory therapy. However, SCIG has the potential to play a more important role in indications besides PI, such as neuropathies and myopathies. Here, the authors review emerging developments in the use of SCIG.

\section{METHODS}

Studies presented here were selected for their contribution to the development of SCIG therapy beyond the established clinical practice, based on the authors' experience in the field. An initial PubMed search was performed using the terms immunoglobulin, immune globulin, subcutaneous, PI, chronic inflammatory demyelinating polyradiculoneuropathy (CIDP), multifocal motor neuropathy (MMN), myasthenia gravis, Kawasaki disease, immune thrombocytopenia, and Guillain-Barré syndrome, without restriction to publication date or publication language.

\section{SCIG IN INITIATION OF REPLACEMENT THERAPY}

Traditionally, maintenance replacement SCIG therapy is preceded by a switch from existing IVIG therapy. Different regimens for initiating SCIG have been tested, but usually the first subcutaneous infusion is given 1 week after the last intravenous infusion in order to maintain high serum IgG levels. ${ }^{13-15}$ Thereafter, the 
average daily IgG level achieved with IVIG can be maintained with regular subcutaneous infusions (Figure 1). Alternatively, however, the IgG loading can be achieved directly with SCIG.

\section{Study of SCIG in Previously Untreated Patients with PI}

A recently completed open-label, single-arm, phase 2 study of Vivaglobin ${ }^{\circledR}$ (CSL Behring GmbH, Marburg, Germany), a 16\% SCIG, in previously untreated patients with PI, showed that initial IgG loading of patients can be easily achieved with daily SCIG administration. ${ }^{16}$

Figure 1. Schematic presentation of serum IgG levels achieved with intravenous and/or subcutaneous administration. Serum IgG levels are presented schematically to illustrate the different rate of $\operatorname{IgG}$ increase with different administration routes and regimens. The curves labeled IVIG and SCIG refer to treatment with IVIG or SCIG alone, without loading. The shaded area marked "Higher risk zone between two IVIG infusions" denotes the waning period of treatment effect, resulting in increased rate of infections in PI or deteriorating muscle strength in $\mathrm{MMN}$. IgG=immunoglobulin G; IVIG=intravenous immunoglobulin; $\mathrm{MMN}=$ multifocal motor neuropathy; $\mathrm{PI}=$ primary immunodeficiency; SCIG=subcutaneous immunoglobulin.

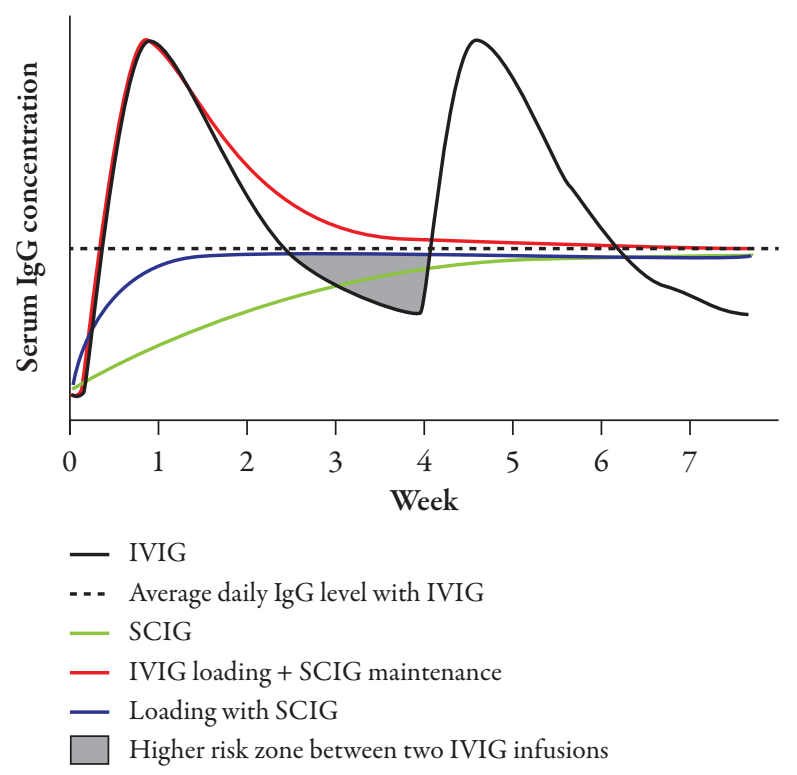

Eighteen patients (aged 2-65 years) received an initial loading dose of $100 \mathrm{mg} / \mathrm{kg}$ body weight/day for 5 consecutive days followed by maintenance therapy with $100-200 \mathrm{mg} / \mathrm{kg}$ weekly (Figure 2A). Seventeen patients (94\%) achieved the target serum IgG level of $\geq 5 \mathrm{~g} / \mathrm{L}$ by day 12 ( 1 week after completion of the loading dose) and one patient achieved the target IgG level by day 26. Mean IgG levels increased more than twofold from screening to day 12 (Figure $2 \mathrm{~B})^{16}$ and remained stable for the entire 6-month maintenance phase of the study. The study design allowed dose adjustments in week 3; however, no patient required dose adjustment. The doses chosen at study start were maintained throughout the study and were effective in all patients.

Treatment was well tolerated, with $98 \%$ of AEs being mild or moderate. Similar tolerability has been reported in PI patients switched from IVIG to Vivaglobin in another study: in 60 patients, of whom 16 were children, $98 \%$ of local reactions and $86 \%$ of subcutaneous infusion-related systemic AEs were mild, with only one severe systemic AE (hypotension). ${ }^{14}$

The results from this study showed that protective IgG levels are achieved by initiating SCIG treatment directly, without prior IVIG loading, creating new treatment possibilities for patients with PI.

\section{SCIG in Immunomodulation}

Immunoglobulin treatment is considered the first choice of therapy in a number of autoimmune or inflammatory diseases. A recent report of the United Kingdom (UK) National Immunoglobulin Database identified idiopathic/autoimmune thrombocytopenia as the major hematological indication in which immunoglobulin has been used in the UK between 2008-2009. ${ }^{17}$ In neurological indications, 
Figure 2. Initialization of SCIG therapy in previously untreated primary immunodeficiency (PI) patients. (A) PI study design. Dose adjustments in patients not achieving serum IgG levels of $\geq 5 \mathrm{~g} / \mathrm{L}$ by day 12 were planned for day 15 (an additional dose of $150 \mathrm{mg} / \mathrm{kg} \mathrm{bw}$ ) and day 19 (a dose increased to $150 \mathrm{mg} / \mathrm{kg}$ ). Reproduced from Borte M et al., ${ }^{16} \mathrm{~J} \mathrm{Clin}$ Immunol 2011; Sep 20. [Epub ahead of print] (Fig. 1), with kind permission from Springer Science+Business Media B.V.

(B) Increase in serum IgG levels after five consecutive daily doses of $100 \mathrm{mg} / \mathrm{kg}$ SCIG. Mean \pm SD serum levels are shown. The arrow indicates the target for primary endpoint: $\operatorname{IgG}$ levels $>5 \mathrm{~g} / \mathrm{L}$ at day 12 . bw=body weight; $\operatorname{IgG}=$ immunoglobulin $\mathrm{G}$; s.c.=subcutaneous; $\mathrm{SCIG}=$ subcutaneous immunoglobulin.

A.

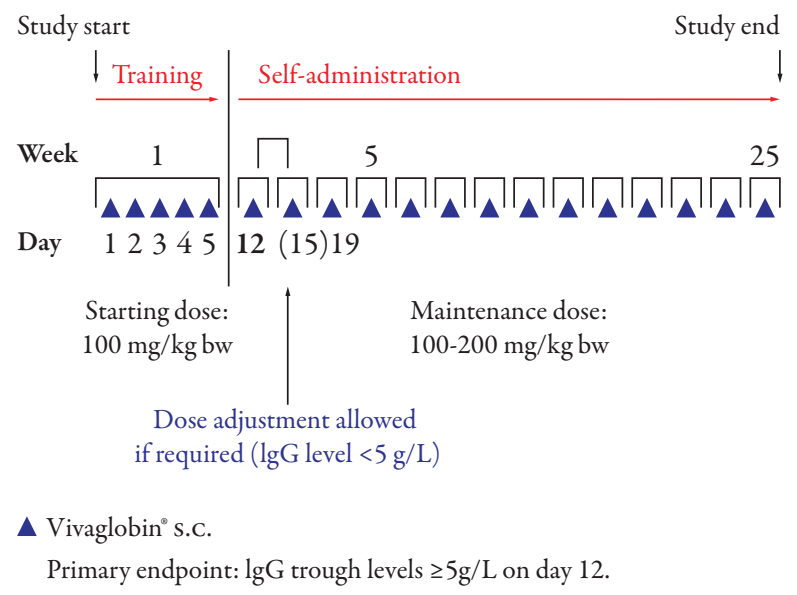

$85 \%$ of immunoglobulin use has been reported in CIDP, MMN, myasthenia gravis, or Guillain-Barré syndrome. ${ }^{17}$ In the recently initiated Assessment of Immunoglobulins in a Long-Term NonInterventional Study (SIGNS), the use of IVIG and SCIG in immunodeficiencies and neurological autoimmune indications will be evaluated. ${ }^{18}$

An updated summary of mechanisms of action and indications for use of immunoglobulin therapy in immunomodulation have been published recently. ${ }^{19}$ It is not clear which of the many immunomodulatory mechanisms of IgG are responsible for its effects in neuropathies or myopathies. ${ }^{20,21}$ Effective immunomodulation is traditionally associated with high IgG doses, although these are not based on actual dosefinding studies. For most conditions, it has been assumed that the dose used in Kawasaki syndrome and immune thrombocytopenia $(2 \mathrm{~g} / \mathrm{kg})$ is needed. It is unknown whether
B.

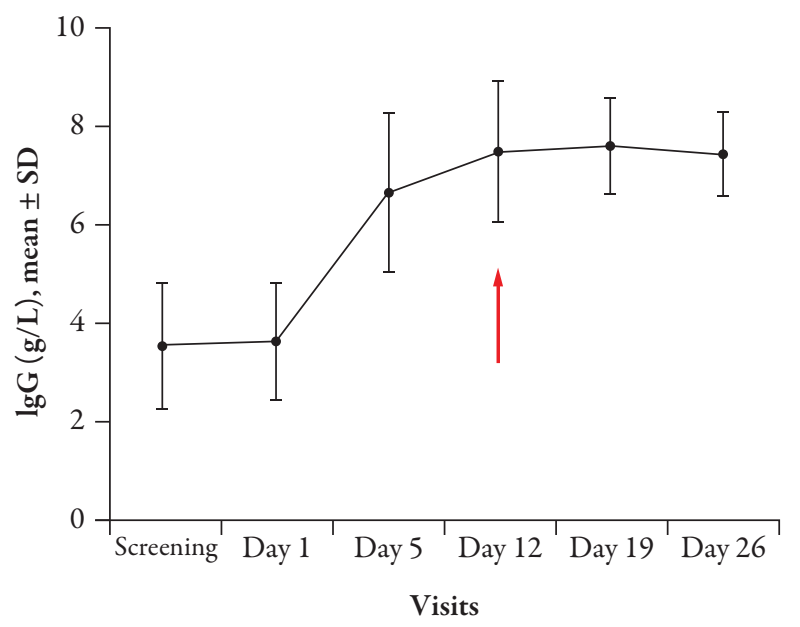

high peaks are necessary for treatment effect, but in several conditions - particularly in neuromuscular diseases - patients experience recurrent symptoms (muscle weakness) at low trough levels when the next intravenous infusion is due. ${ }^{22}$ Studies involving several indications have been initiated to determine whether equivalent-dose SCIG could be as effective as IVIG; eliminating these low troughs and the attendant increase in symptoms. ${ }^{23-29}$

The use of SCIG instead of IVIG in maintenance therapy in MMN, polymyositis, and dermatomyositis has been reported recently. ${ }^{30-34}$

\section{Crossover study of SCIG and IVIG in MMN}

In a randomized, single-blinded, crossover study, nine MMN patients who showed a good response to previous IVIG therapy were enrolled. Responsiveness to IgG therapy was defined as a 
decrease of $\geq 10 \%$ in combined dynamometric strength of one or more muscle groups in a prestudy, treatment-free period of a maximum 10 weeks. ${ }^{32}$ Prior to entering the main study, muscle strength was restored by administering two IVIG doses as a "wash-out" treatment prior to the main study. In the main study, patients were randomized to receive SCIG (Subcuvia ${ }^{\circledR}$, Baxter International Inc., Deerfield, IL, USA) or IVIG (Endobulin ${ }^{\circledR}$, Baxter International Inc.) for a period equivalent to three IVIG treatment intervals (18-56 days) and then switched to the other treatment. SCIG was administered two to three times weekly, while IVIG was given at individually adjusted intervals. The two treatments were equally effective and the combined dynamometric strength was maintained in eight of nine patients during each intervention period; one patient was poorly compliant. Thus, SCIG was as effective as IVIG in short-term treatment. Transient injection site reactions during SCIG treatment were reported by six patients, but only one patient experienced sustained erythema and edema at the injection site that necessitated temporary reduction of the injected volume. Three patients on IVIG reported AEs: rash, phlebitis, and venous catheter infection. ${ }^{32}$

\section{Dose-Finding Study of SCIG in MMN}

In a single-center, open-label study, 10 patients with $\mathrm{MMN}$ were treated one to two times a week for 6 months with SCIG (GammaQuin ${ }^{\circledR}$, Sanquin, Amsterdam, The Netherlands) at monthly doses equivalent to either $50 \%$ or $100 \%$ of the previous IVIG dose (five patients in each group) ${ }^{31}$ In case of worsening of disease symptoms, the low dose could be doubled. The primary endpoint was muscle strength in 10 predefined bilateral muscle groups, evaluated using the Medical Research Council Scale.
In the low-dose group, one patient discontinued the study due to injection site reactions (local swelling and pain) and the remaining four patients experienced deteriorating muscle strength and had to be reloaded with IVIG, which resulted in improvement. Four of five patients in the equivalent-dose group maintained muscle strength throughout the study. The fifth patient was administered an IVIG loading dose and, because of the patient's preference for an SCIG treatment, maintained on a higher SCIG dose (166\% of the previous IVIG dose) with which muscle strength remained stable. The treatment was tolerated well, with no serious AEs and decreasing incidence of local reactions during therapy.

\section{Smooth Transition Protocol Study of SCIG in MMN}

A recently completed prospective, open-label, multicenter, phase 2 study in patients with MMN showed that IgG concentrations can be maintained over 6 months with weekly SCIG (Vivaglobin) administration using a protocol in which the dose of SCIG was increased weekly to maintain the serum IgG levels achieved with prior IVIG therapy (smooth transition protocol). ${ }^{34}$ After an initial run-in period, eight patients aged 42-66 years on stable IVIG treatment received weekly subcutaneous Vivaglobin infusions for 24 weeks (Figure 3A) ${ }^{34}$ at doses equivalent to the calculated weekly IVIG dose from previous therapy. The dose was adjusted stepwise during the first month to achieve a smooth transition of the total administered IgG dose from monthly IVIG treatment to weekly SCIG infusions. With a mean dose of $272 \mathrm{mg} / \mathrm{kg}$ /week (corresponding to $1087 \mathrm{mg} / \mathrm{kg} / \mathrm{month}$ ), seven of eight patients maintained serum IgG levels (14-22 g/L) similar to those at study start and had stable or 
Figure 3. Maintenance therapy with SCIG in MMN patients. (A) MMN study design. Dose increases of $25 \%$ in patients with deteriorating muscle strength were planned for week 8 or 16. Reproduced from Misbah S et al., ${ }^{34} \mathrm{~J}$ Peripher Nerv Syst 2011;16:92-97 (Fig. 1), with kind permission from John Wiley \& Sons Ltd. (B) Maintenance of muscle strength. Muscle strength scores at baseline and week 24 are shown. The strengths of 40 standardized muscles or muscle groups of the upper and lower limbs (20 on each side) were assessed according to the MRC Scale. The full scale ranges from 0-200 points, with 200 meaning normal muscle power. Patient 2 (red cross) discontinued at week 12 due to progressive worsening despite dose increase. (C) Clinical scores as function of IgG trough levels in one patient who discontinued due to treatment failure. Worsening in muscle strength, disability score and motor function score, in the MMN patient who discontinued due to treatment failure is shown together with serum IgG concentrations (IgG and motor function data available only for baseline and week 8 due to discontinuation after week 12). Muscle strength score was determined as described for Figure 3B. Disability was assessed using a modified Guy's Neurological Disability scale. The scale ranges from 0-10 points, with 10 meaning inability to use arms and legs. Motor function score was based on 4 individually defined tasks. The scale ranges from 0-16 points, with 16 meaning inability to perform any task. IVIG=intravenous immunoglobulin; $\mathrm{MMN}=$ mutifocular motor neuropathy; MRC=Medical Research Council; s.c.=subcutaneous; SCIG=subcutaneous immunoglobulin.

A.

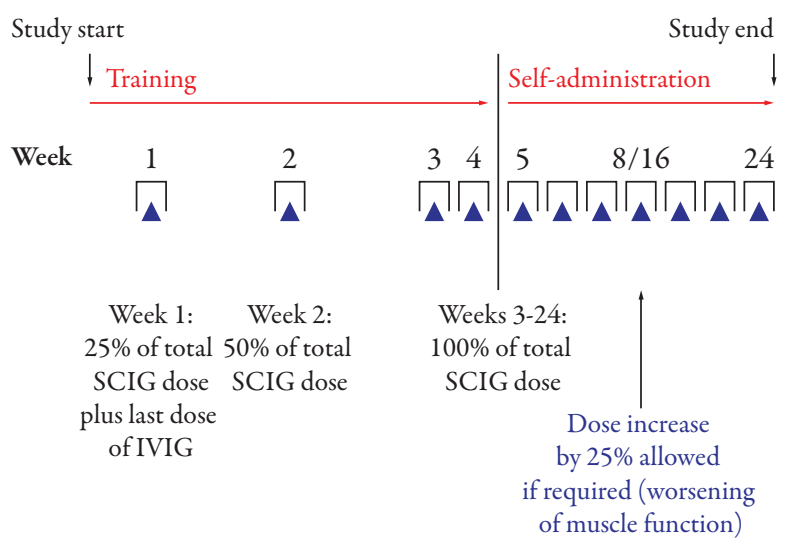

$\Delta$ Vivaglobin s.c.

Primary endpoint: muscle strength at Week 24 with SCIG comparable to that with previous IVIG.
B.

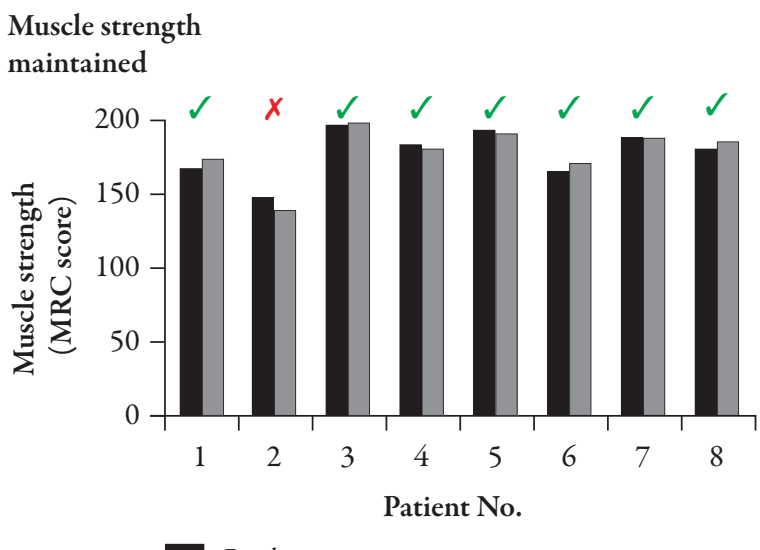

Baseline

Week 24

C.

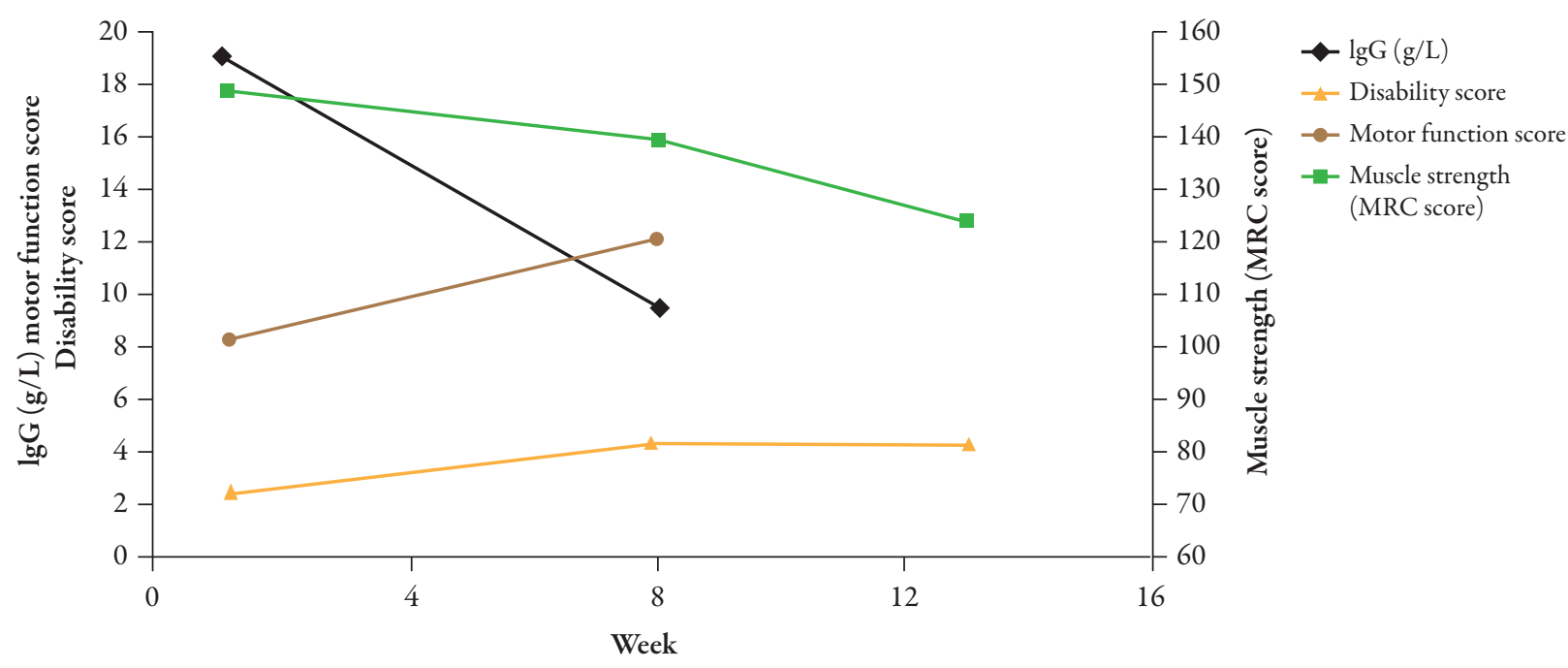


somewhat improved muscle strength (Figure 3B). One patient's muscle strength and disability worsened despite dose increase, resulting in withdrawal from the study (Figure 3C). This patient had a body mass index indicative of cachexia $\left(<17.5 \mathrm{~kg} / \mathrm{m}^{2}\right)$, suggesting that IgG absorption from the subcutaneous sites may have been suboptimal. Patients felt comfortable with self-infusion and tolerated SCIG administration well. Four patients did not experience any AEs. No severe AEs were reported and $89 \%$ of all AEs were mild. Only one patient experienced injection site reactions (edema, pruritus, and skin reaction). The results of this study suggest that rapidly raising peak IgG concentrations, as achieved with intravenous administration, may not be required for ongoing clinical efficacy in MMN. Due to the small number of patients in the study, the results need to be verified and expanded to other diseases requiring immunomodulatory therapy.

\section{SCIG Therapy in Other Neuropathies}

Two case reports of the use of SCIG in the maintenance of CIDP demonstrated stabilization of patients with monthly doses equivalent to previous IVIG treatment. ${ }^{33}$ The weekly dose was administered either once per week or on three consecutive days per week. Tolerability was good, with only local reactions observed.

Initiation of immunomodulation therapy with SCIG in patients with polymyositis or dermatomyositis was reported recently. ${ }^{30}$ Although six of the seven patients had been treated with IVIG at some point, four were not receiving IVIG at the start of the study. In these patients, SCIG therapy was initiated by once weekly administration of $0.2 \mathrm{~g} / \mathrm{kg} /$ week, resulting in improved serum creatine kinase levels and muscle strength. ${ }^{30}$ Apart from mild local reactions in two patients, no major AEs were observed.

\section{DISCUSSION}

\section{The Importance of Stable Trough IgG Levels}

Despite the small patient populations in the studies described above, it appears that both intravenous and subcutaneous regimens can be used in the initiation of replacement therapy in PI and maintenance of patients with MMN.

It has been suggested that a minimal IgG concentration $(5 \mathrm{~g} / \mathrm{L})$ is required for protection from infections in immunodeficiencies ${ }^{35-37}$ and that higher serum IgG levels result in better protection. ${ }^{38-40}$ Thus, achievement of an optimal serum IgG level has become a primary target of therapy. ${ }^{41}$ Patient databases, such as the European Society for Immunodeficiencies (ESID) online registry, clearly suggest improved efficacy at higher serum IgG levels. For example, in patients with common variable immunodeficiency, infection rate and number of days spent in hospital decrease substantially as IgG levels increase from $<5 \mathrm{~g} / \mathrm{L}$ to $>7 \mathrm{~g} / \mathrm{L} .{ }^{41}$ In two recent studies of IgPro20 (Hizentra ${ }^{\circledR}$, CSL Behring, Berne, Switzerland) in PI, using median IgG doses of 113.9 and $213.2 \mathrm{mg} / \mathrm{kg}$, 22,43 mean IgG trough levels of 8.10 and $12.53 \mathrm{~g} / \mathrm{L}$, respectively, were achieved. There were no serious infections and the corresponding rates of non-serious infections were 5.18 versus 2.76 infections/patient/year, respectively.

The correlation between IgG levels and clinical outcomes in $\mathrm{MMN}$ is illustrated with data for one patient from the smooth transition protocol study, whose IgG levels failed to increase, probably due to the low body fat, with associated worsening of disease symptoms (see Figure 3C). ${ }^{34}$ It remains to be established whether stable IgG trough levels are as effective in autoimmune-mediated disorders as they are in PI. After the pioneering work of Imbach et al. in immune thrombocytopenia, ${ }^{44}$ 
clinicians have used similar high doses of IVIG, without dose ranging or alternative treatment schedule studies, for the successful treatment of other disorders with autoimmune and/or inflammatory pathogenesis.

\section{Active Choice for Patients}

With increasingly widespread use of SCIG, patients have the opportunity to choose a treatment schedule to fit a lifestyle, family activities, and personal preference. Physicians should take into account both clinical and lifestyle factors when selecting the route of administration (Table 1). Important clinical factors are venous access and the tolerability of the IgG product used. ${ }^{45}$ Particular emphasis should be placed on the timely and thorough patient characterization to ensure that risk factors for AEs are identified before treatment with IVIG or SCIG is chosen. Underlying conditions predisposing to acute renal insufficiency after IVIG administration have been adequately described and may include renal insufficiency, diabetes mellitus, volume depletion, sepsis, paraproteinemia, high titer of rheumatoid factor, and advanced age. ${ }^{46}$ High serum viscosity may lead to vascular events such as thromboembolism and a history of migraine has been associated with an increased risk of aseptic meningitis after IVIG. ${ }^{46-48}$

Patients' attitudes to IVIG and SCIG are based on both preferences and concerns. Patients who live at a distance from doctor's office or infusion center, as well as those who are employed or travel frequently usually prefer to infuse at home. Patients making this choice are willing to complete the training and take responsibility for the treatment. A preference for clinic or infusion center IVIG therapy is observed among the elderly, the unemployed, those with aversion to needles, and those with fear of facing potential
AEs at home. ${ }^{11,12,49,50}$ Some patients prefer home therapy regardless of the administration route ${ }^{11}$ and in that case SCIG therapy may often be more appropriate. The safety and security of the patient's home environment, the patient's schedule and availability during business hours, and the level of support the patient receives from family and physician are crucial factors in decision making. In addition, selecting one route of administration over the other must take into consideration the different AE profiles of IVIG and SCIG products: IVIG is more frequently associated with systemic AEs, such as headache, nausea, and fatigue; ${ }^{51}$ SCIG administration is largely free of these AEs, but may cause infusion site reactions, such as swelling and redness. ${ }^{36}$

The treatment options available in clinical practice are extremely diverse. The same IgG dose can be administered in several different ways depending on the patient's preference. Slow overnight administration, fast infusion with two pumps (less than 1 hour), and frequent, rapid manual (push) infusions all are viable options. With the frequent push method, a volume of 3-20 mL of Vivaglobin can be easily administered daily or on alternate days without the cost or complications of a mechanical pump..$^{21,22}$

\section{New Products Allow Faster Subcutaneous Infusions}

Preparations for intramuscular immunoglobulin (IMIG) administration were the first products to be infused subcutaneously. ${ }^{8,9,52}$ Infusions were initially very slow (10-20 mL over several hours), but as the improved tolerability of SCIG was recognized, infusion rates were increased. ${ }^{3,11,53}$ Currently available 16\% products are infused at 10-20 mL (1.6-3.2 g) per hour (Table 2). ${ }^{14,15}$ The recent introduction of a $20 \%$ product (Hizentra), specifically formulated for subcutaneous use, allowed even higher 
Table 1. Key features of intravenous immunoglobin (IVIG) and subcutaneous immunoglobin (SCIG).

\begin{tabular}{lll}
\hline & IVIG & SCIG \\
\hline Efficacy & & \\
Stable serum IgG levels & No & Yes \\
Peak serum IgG & Yes & No \\
Protection from infections & Yes & Yes \\
\hline
\end{tabular}

\section{Tolerability}

Treatment-associated systemic adverse events Yes

Treatment-related local reactions

\section{Administration specifics}

Need for venous access

Duration of infusion

Frequency of infusions

Yes

None or very rare

None or very rare

Yes

Yes

Several hours

Once every 2-4 weeks

\section{Convenience}

Self-infusion

Home administration

Training for home therapy

Flexibility for the patients Yes (but more technically demanding)

Yes (but now less frequently chosen) Yes (preferred by most patients)

Yes (longer and more technically demanding, need for good veins)

Yes (typically achieved during first

Yes (2-4 weekly administrations are necessary)

Suitable for active life style (employment, school, Yes (chosen by some patients because of the longer gap between treatments)

\section{Indications}

Immunodeficiencies ("replacement-dose” Yes

Yes indications)

Autoimmune or inflammatory conditions Yes ("high-dose" indications)

Initiation of immunoglobulin therapy

Yes

Maintenance immunoglobulin therapy

Yes
Yes (preferred by most patients, especially children) 3-4 training sessions

No

Typically 1 hour or less

Typically once a week (ranges from once a day to once every 2 weeks)

Yes (once daily, 2-4 times per week, once weekly, once every 2 weeks; pump or push infusion)

Yes sports, frequent travel, etc)

Yes, increasingly used in neurological indications

Yes, evaluated in primary

immunodeficiencies

Yes

$\mathrm{IgG}=$ immunoglobulin $\mathrm{G}$. 


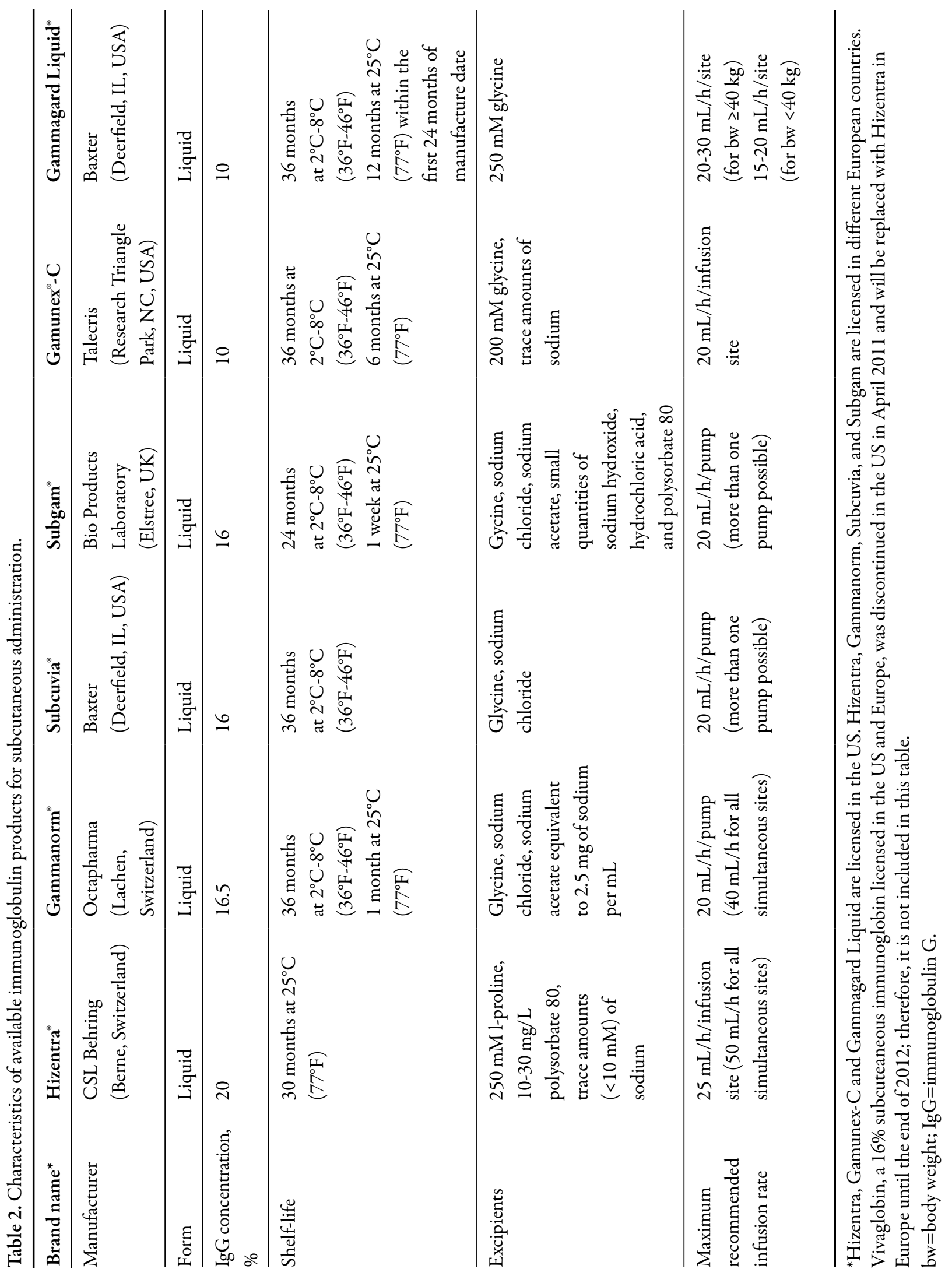


flow rates: up to $50 \mathrm{~mL}(10 \mathrm{~g})$ per hour ${ }^{42}$ or $70 \mathrm{~mL}$ (14 g) per hour ${ }^{43}$ without compromising local tolerability in two phase 3 trials.

Other tools for optimization are based on techniques previously used in the infusion of other subcutaneous medications. Hyaluronidase of animal origin has been used to increase tissue permeability by partial degradation of the extracellular matrix, which is a significant barrier to subcutaneous delivery. The development of recombinant human hyaluronidase (rHuPH20) has also opened new possibilities for IgG administration. ${ }^{21,54,55}$ Implanted subcutaneous infusion ports may help the infused fluid to distribute more evenly into the tissue; thus, potentially allowing increased volumes and shortened infusion times. However, with any implanted device in the setting of immunodeficiency, the risk of infection would need to be considered.

\section{Subcutaneous Administration and Home Therapy Reduce the Cost of Immunoglobulin Treatment}

Several studies have compared the costs of IgG treatment with subcutaneous versus intravenous and home versus hospital/office administration. The total yearly cost of home therapy was found to be $50 \%$ of the cost of hospital-based therapy in Sweden, Denmark, and Norway, and the out-of-pocket costs of patients were reduced by $85 \% .{ }^{53}$ However, the reduction in costs was partially due to the substantially lower price of the IMIG used for the subcutaneous route. In Germany, SCIG treatment was found to be less expensive than IVIG treatment by approximately $50 \%$ due to the substantially reduced costs for treatment and diagnostic procedures, as well as the time for sick leave of caregivers of pediatric patients. ${ }^{56} \mathrm{~A}$ therapyrelated cost reduction of $50 \%$ was reported in two case studies of patients with CIDP who switched from IVIG to SCIG receiving equivalent doses. ${ }^{33} \mathrm{~A}$ study performed by the Canadian Government Agency for Drugs and Technologies in Health (CADTH) provided data showing that home administration of IVIG or SCIG in patients with PI in Canada would be more cost-effective than hospital/office administration: the overall costs for self-administered IVIG (100\%) and selfadministered SCIG (103.6\%) were lower than the costs for conventional hospital-administered IVIG therapy (113.5\%). ${ }^{57}$ Thus, there remain potential savings that are independent of route, home administration, and drug costs.

More pharmacoeconomic studies in Europe and the USA will be needed to evaluate the costs of immunoglobulin treatment, including products, healthcare personnel, hospital and facility charges, and infusion equipment, which add to the overall costs of treatment. ${ }^{58}$ These studies should take into consideration the value to the patient and family of costs now required for travel to the office or clinic, and time spent waiting for IVIG administration. The current clinical practice gives the general perception that home therapy and/or self-infusion are cost-neutral in most countries, but offer the added benefit of improvements to patients' quality of life. ${ }^{12,59}$ Additional potential sources of cost reduction with optimal immunoglobulin replacement therapy are the improved treatment efficacy and good tolerability of the currently used products, resulting in lower costs for the treatment of disease-specific symptoms (eg, recurrent infections in patients with PI), maintenance of the ability to ambulate and conduct activities of daily living (in neuropathies/ myopathies), and alleviation or treatment of AEs. A potential source of increased cost for SCIG therapy in the US would be the current Food and Drug Administration (FDA) recommendation to use higher doses than the previous IVIG dose. 


\section{CONCLUSION}

A number of IgG treatment options are now available, with the development of high-quality new products for intravenous and subcutaneous administration. SCIG therapy appeals to many patients with its simple integration into everyday life, shorter training period for home therapy, and flexibility. The potential applicability of SCIG for indications beside PI, such as neuropathies, myopathies, and autoimmune or inflammatory disorders should allow it to play a more prominent role in areas currently reserved only for IVIG. Patients are increasingly aware of the treatment possibilities for a disease and are more willing to take responsibility for the treatment in exchange for flexibility. Improving choice amongst treatment options requires ongoing support from physicians and nurses in patient education and training, improving the availability of equipment and healthcare personnel, and providing a flexible individualized package of care for patients.

\section{ACKNOWLEDGMENTS}

No funding was used for the preparation of this manuscript. The opinions expressed in this paper are those of the authors. The authors thank Dr. Othmar Zenker for help during the conduct of the studies and Dr. Andrea Sebald, Dr. Martin Bexon, and Dr. Jeff Baggish for critical review of the manuscript. Editorial assistance in the preparation of this manuscript was provided by Dr. Emiliana Jelezarova of Phocus Services Ltd. Support for this assistance was funded by CSL Behring. S.J. participated in preparation of the manuscript; M.R.S. in critical revision of the manuscript; H.J.L. in critical revision of the manuscript; M.B. in preparation of the manuscript; B.R. in critical revision of the manuscript; M.H.S. in preparation of the manuscript; M.B. in preparation of the manuscript. S.J. is the guarantor for this article, and takes responsibility for the integrity of the work as a whole. S.J. reports clinical trial support from CSL Behring, Baxter, and Octapharma, and consultant fees from Baxter, Octapharma, CSL Behring, and Biotest; M.R.S. reports clinical trial support from CSL Behring, Baxter, Biotest Pharmaceuticals, and Bio Product Laboratory; H.J.L. reports funding to attend conferences and other educational events, donations to her departmental fund and/ or clinical trial support from BPL, CSL Behring, Octapharma, Baxter, and Grifols, member of the medical advisory panel for Baxter and CSL Behring; M.B. reports consultant fees from CSL Behring and Baxter, and honoraria for lectures from CSL Behring, Baxter, and Octapharma; B.R. reports clinical trial support from CSL Behring, Baxter, Wyeth, and Novo Nordisk; M.H.S. has no conflicts of interest; M.B. is an employee of CSL Behring LLC, King of Prussia, PA, USA, with equity interests, and Adjunct Professor of Pediatrics and Pathology at Case Western Reserve University in Cleveland, Ohio, USA. No other potential conflicts of interest were reported.

Open Access. This article is distributed under the terms of the Creative Commons Attribution Noncommercial License which permits any noncommercial use, distribution, and reproduction in any medium, provided the original authors and source are credited.

\section{REFERENCES}

1. Bruton OC. Agammaglobulinemia. Pediatrics. 1952;9:722-728.

2. Berger M, Cupps TR, Fauci AS. Immunoglobulin replacement therapy by slow subcutaneous infusion. Ann Intern Med. 1980;93:55-56.

3. Gardulf A, Hammarstrom L, Smith CI. Home treatment of hypogammaglobulinaemia with subcutaneous gammaglobulin by rapid infusion. Lancet. 1991;338:162-166. 
4. Hansen S, Gustafson R, Smith CI, Gardulf A. Express subcutaneous IgG infusions: decreased time of delivery with maintained safety. Clin Immunol. 2002;104:237-241.

5. Gardulf A. Immunoglobulin treatment for primary antibody deficiencies: advantages of the subcutaneous route. BioDrugs. 2007;21:105-116.

6. Moore ML, Quinn JM. Subcutaneous immunoglobulin replacement therapy for primary antibody deficiency: advancements into the 21st century. Ann Allergy Asthma Immunol. 2008;101:114-121.

7. Abrahamsen TG, Sandersen H, Bustnes A. Home therapy with subcutaneous immunoglobulin infusions in children with congenital immunodeficiencies. Pediatrics. 1996;98:1127-1131.

8. Roord JJ, van der Meer JW, Kuis W, et al. Home treatment in patients with antibody deficiency by slow subcutaneous infusion of gammaglobulin. Lancet. 1982;1:689-690.

9. Ugazio AG, Duse M, Re R, Mangili G, Burgio GR. Subcutaneous infusion of gammaglobulins in management of agammaglobulinaemia. Lancet. 1982;1:226-227.

10. Daly PB, Evans JH, Kobayashi RH, et al. Homebased immunoglobulin infusion therapy: quality of life and patient health perceptions. Ann Allergy. 1991;67:504-510.

11. Gardulf A, Nicolay U, Math D, et al. Children and adults with primary antibody deficiencies gain quality of life by subcutaneous IgG self-infusions at home. J Allergy Clin Immunol. 2004;114:936-942.

12. Nicolay U, Kiessling $P$, Berger $M$, et al. Healthrelated quality of life and treatment satisfaction in North American patients with primary immune deficiency diseases receiving subcutaneous IgG selfinfusions at home. J Clin Immunol. 2006;26:65-72.

13. Desai SH, Chouksey A, Poll J, Berger M. A pilot study of equal doses of $10 \%$ IGIV given intravenously or subcutaneously. J Allergy Clin Immunol. 2009;124:854-856.

14. Gardulf A, Nicolay U, Asensio O, et al. Rapid subcutaneous IgG replacement therapy is effective and safe in children and adults with primary immunodeficiencies - a prospective, multinational study. J Clin Immunol. 2006;26:177-185.

15. Ochs HD, Gupta S, Kiessling P, Nicolay U, Berger M. Safety and efficacy of self-administered subcutaneous immunoglobulin in patients with primary immunodeficiency diseases. J Clin Immunol. 2006;26:265-273.

16. Borte M, Quinti I, Soresina A, et al. Efficacy and safety of subcutaneous Vivaglobin ${ }^{\circledR}$ replacement therapy in previously untreated patients with primary immunodeficiency: a prospective, multicenter study. J Clin Immunol. 2011. [Epub ahead of print]

17. Sewell C. First national immunoglobulin database report (2008-2009). London: Department of Health, National Health Service, London, UK; 2010. Available at: http://www.ivig.nhs.uk/ documents/DH_ImmunoDatabaseReport\%202010. pdf. Accessed on November 14, 2011

18. Kirch W, Gold R, Hensel M, et al. Assessment of immunoglobulins in a long-term noninterventional study (SIGNS Study). Rationale, design, and methods. Med Klin (Munich). 2010;105:647-651.

19. Rezaei N, Abolhassani H, Aghamohammadi A, Ochs HD. Indications and safety of intravenous and subcutaneous immunoglobulin therapy. Expert Rev Clin Immunol. 2011;7:301-316.

20. Hughes RA, Dalakas MC, Cornblath DR, Latov N, Weksler ME, Relkin N. Clinical applications of intravenous immunoglobulins in neurology. Clin Exp Immunol. 2009;158(Suppl. 1):34-42.

21. Misbah S, Sturzenegger MH, Borte M, et al. Subcutaneous immunoglobulin: opportunities and outlook. Clin Exp Immunol. 2009; 158(Suppl. 1):51-59.

22. Berger M. Subcutaneous IgG replacement in immune deficiency diseases. Clinical focus on primary immune deficiencies. Immune Deficiency Foundation. 2008;13:1-8.

23. Dalakas MC, Illa I, Dambrosia JM, et al. A controlled trial of high-dose intravenous immune globulin infusions as treatment for dermatomyositis. N Engl J Med. 1993;329:19932000.

24. Eftimov F, Winer JB, Vermeulen $M$, de Haan $\mathrm{R}$, van Schaik I. Intravenous immunoglobulin for chronic inflammatory demyelinating polyradiculoneuropathy. Cochrane Database Syst Rev. 2009;CD001797.

25. Gajdos P, Chevret S, Toyka K. Intravenous immunoglobulin for myasthenia gravis. Cochrane Database Syst Rev. 2003;CD002277. 
26. Hughes RA, Raphael JC, Swan AV, van Doorn PA. Intravenous immunoglobulin for GuillainBarre syndrome. Cochrane Database Syst Rev. 2006;CD002063.

27. Hughes RA, Donofrio P, Bril V, et al. Intravenous immune globulin (10\% caprylate-chromatography purified) for the treatment of chronic inflammatory demyelinating polyradiculoneuropathy (ICE study): a randomised placebo-controlled trial. Lancet Neurol. 2008;7:136-144.

28. Levy Y, Uziel Y, Zandman G, et al. Response of vasculitic peripheral neuropathy to intravenous immunoglobulin. Ann N Y Acad Sci. 2005;1051:779-786.

29. van Schaik IN, van den Berg LH, de Haan R, Vermeulen M. Intravenous immunoglobulin for multifocal motor neuropathy. Cochrane Database Syst Rev. 2005;CD004429.

30. Danieli MG, Pettinari L, Moretti R, Logullo F, Gabrielli A. Subcutaneous immunoglobulin in polymyositis and dermatomyositis: a novel application. Autoimmun Rev. 2011;10:144-149.

31. Eftimov F, Vermeulen M, de Haan RJ, van den Berg LH, van Schaik IN. Subcutaneous immunoglobulin therapy for multifocal motor neuropathy. J Peripher Nerv Syst. 2009;14:93-100.

32. Harbo T, Andersen H, Hess A, Hansen K, Sindrup $\mathrm{SH}$, Jakobsen J. Subcutaneous versus intravenous immunoglobulin in multifocal motor neuropathy: a randomized, single-blinded cross-over trial. Eur J Neurol. 2009;16:631-638.

33. Lee DH, Linker RA, Paulus W, Schneider-Gold C, Chan A, Gold R. Subcutaneous immunoglobulin infusion: a new therapeutic option in chronic inflammatory demyelinating polyneuropathy. Muscle Nerve. 2008;37:406-409.

34. Misbah S, Baumann A, Fazio R, et al. A smooth transition protocol for patients with multifocal motor neuropathy going from intravenous to subcutaneous immunoglobulin therapy: an openlabel proof-of-concept study. J Peripher Nerv Syst. 2011;16:92-97.

35. Bonagura VR, Marchlewski R, Cox A, Rosenthal DW. Biologic IgG level in primary immunodeficiency disease: the IgG level that protects against recurrent infection. J Allergy Clin Immunol. 2008;122:210-212.

36. Orange JS, Hossny EM, Weiler CR, et al. Use of intravenous immunoglobulin in human disease: a review of evidence by members of the Primary Immunodeficiency Committee of the American Academy of Allergy, Asthma and Immunology. J Allergy Clin Immunol. 2006;117:S525-S553.

37. Stiehm ER. Human intravenous immunoglobulin in primary and secondary antibody deficiencies. Pediatr Infect Dis J. 1997;16:696-707.

38. Eijkhout HW, van der Meer JW, Kallenberg CG, et al. The effect of two different dosages of intravenous immunoglobulin on the incidence of recurrent infections in patients with primary hypogammaglobulinemia. A randomized, doubleblind, multicenter crossover trial. Ann Intern Med. 2001;135:165-174.

39. Lucas M, Hugh-Jones K, Welby A, Misbah S, Spaeth P, Chapel H. Immunomodulatory therapy to achieve maximum efficacy: doses, monitoring, compliance, and self-infusion at home. J Clin Immunol. 2010;30(Suppl. 1):S84-S89.

40. Orange JS, Grossman WJ, Navickis RJ, Wilkes MM. Impact of trough IgG on pneumonia incidence in primary immunodeficiency: a meta-analysis of clinical studies. Clin Immunol. 2010;137:21-30.

41. Ballow M, Notarangelo L, Grimbacher B, et al. Immunodeficiencies. Clin Exp Immunol. 2009;158(Suppl. 1):14-22.

42. Hagan JB, Fasano MB, Spector S, et al. Efficacy and safety of a new $20 \%$ immunoglobulin preparation for subcutaneous administration, IgPro20, in patients with primary immunodeficiency. J Clin Immunol. 2010;30:734-745.

43. Jolles S, Bernatowska E, de Gracia J, et al. Efficacy and safety of Hizentra ${ }^{\circledR}$ in patients with primary immunodeficiency after a dose-equivalent switch from intravenous or subcutaneous replacement therapy. Clin Immunol. 2011;141:90-102.

44. Imbach $\mathrm{P}$, Barandun $\mathrm{S}$, Baumgartner $\mathrm{C}$, Hirt A, Hofer F, Wagner HP. High-dose intravenous gammaglobulin therapy of refractory, in particular idiopathic thrombocytopenia in childhood. Helv Paediatr Acta. 1981;36:81-86.

45. Berger $M$. Principles of and advances in immunoglobulin replacement therapy for primary immunodeficiency. Immunol Allergy Clin North Am. 2008;28:413-437.

46. Pierce LR, Jain N. Risks associated with the use of intravenous immunoglobulin. Transfus Med Rev. 2003;17:241-251. 
47. Sekul EA, Cupler EJ, Dalakas MC. Aseptic meningitis associated with high-dose intravenous immunoglobulin therapy: frequency and risk factors. Ann Intern Med. 1994;121:259-262.

48. Dalakas MC. High-dose intravenous immunoglobulin and serum viscosity: risk of precipitating thromboembolic events. Neurology. 1994;44:223-226.

49. Gardulf A, Bjorvell H, Gustafson R, Hammarstrom L, Smith CI. The life situations of patients with primary antibody deficiency untreated or treated with subcutaneous gammaglobulin infusions. Clin Exp Immunol. 1993;92:200-204.

50. Kittner JM, Grimbacher B, Wulff W, Jager B, Schmidt RE. Patients' attitude to subcutaneous immunoglobulin substitution as home therapy. J Clin Immunol. 2006;26:400-405.

51. Tcheurekdjian H, Martin J, Kobayashi R, Wasserman R, Hostoffer R. Intrainfusion and postinfusion adverse events related to intravenous immunoglobulin therapy in immunodeficiency states. Allergy Asthma Proc. 2006;27:532-536.

52. Berger $M$, Cupps TR, Fauci AS. High-dose immunoglobulin replacement therapy by slow subcutaneous infusion during pregnancy. JAMA. 1982;247:2824-2825.

53. Gardulf A, Andersen V, Bjorkander J, et al. Subcutaneous immunoglobulin replacement in patients with primary antibody deficiencies: safety and costs. Lancet. 1995;345:365-369.
54. Frost GI. Recombinant human hyaluronidase (rHuPH20): an enabling platform for subcutaneous drug and fluid administration. Expert Opin Drug Deliv. 2007;4:427-440.

55. Knight E, Carne E, Novak B, et al. Self-administered hyaluronidase-facilitated subcutaneous immunoglobulin home therapy in a patient with primary immunodeficiency. J Clin Pathol. 2010;63:846-847.

56. Hogy B, Keinecke HO, Borte M. Pharmacoeconomic evaluation of immunoglobulin treatment in patients with antibody deficiencies from the perspective of the German statutory health insurance. Eur J Health Econ. 2005;6:24-29.

57. Ho C, Membe S, Cimon K, Roifman C, Kanani A, Morrison A. Overview of subcutaneous versus intravenous immunoglobulin for primary immunodeficiencies: systematic review and economic analysis [Technology overview number 36]. Ottawa: Canadian Agency for Drugs and Technologies in Health. Available at: http://www. cadth ca/index php/en/publication/785. Accessed on November 14, 2011.

58. Berger M. Subcutaneous administration of IgG. Immunol Allergy Clin North Am. 2008;28:779-802

59. Nicolay U, Haag S, Eichmann F, Herget S, Spruck D, Gardulf A. Measuring treatment satisfaction in patients with primary immunodeficiency diseases receiving lifelong immunoglobulin replacement therapy. Qual Life Res. 2005;14:1683-1691. 